\title{
Perfil socioeconômico e nível de satisfação dos pacientes atendidos na clínica de endodontia de uma instituição de pós-graduação
}

\author{
Emmanuelle Cristianne Brandão Riedel*; Marcílio Oliveira Melo**; Erick Thiago de Sousa**; \\ Markelane Santana Silva** \\ * Especialista em Endodontia \\ ** Professor (a), curso de especialização em Endodontia, \\ Associação Brasileira de Odontologia - Secção Piauí
}

Recebido: 25/04/2020. Aprovado: 19/08/2020.

\begin{abstract}
RESUMO
Este estudo objetivou avaliar o perfil socioeconômico e a satisfação dos pacientes em relação aos serviços prestados na Clínica de Endodontia de uma instituição de ensino de pós-graduação. A coleta de dados foi realizada em corte transversal por meio de questionário estruturado e autoaplicável. A amostra foi intencional e aleatória $(n=50)$ para usuários que haviam finalizado o tratamento endodôntico. Para avaliar a satisfação ao final do tratamento foi utilizada escala dimensional e bipolar, com escores atribuídos entre as extremidades. As notas dadas aos diferentes aspectos do tratamento variaram de 0 a 10 com cutoff no valor 7 . Os resultados foram catalogados e submetidos às análises descritiva e inferencial com nível de significância fixado em $5 \%$. O perfil socioeconômico dos pacientes atendidos contempla, em sua maioria, mulheres casadas, pardas, acima da terceira década de vida, com vínculo empregatício e baixa renda familiar, com ensino médio completo, residentes na capital e que usam ônibus e carro próprio como meio de transporte. $\mathrm{O}$ atendimento foi considerado satisfatório. Os usuários procuram o atendimento mais por indicação de outros indivíduos do que pela própria condição financeira, estão satisfeitos com o atendimento do aluno e do professor e consideram a infraestrutura da instituição confortável. Mesmo assim, ainda há aspectos que necessitam ser melhorados, principalmente no que diz respeito ao tempo de espera e de atendimento e à possibilidade atendimento mais de uma vez na semana.

Descritores: Satisfação do Paciente. Avaliação de Serviços de Saúde. Instituições Acadêmicas. Endodontia.
\end{abstract}

\section{INTRODUÇÃO}

As clínicas de pós-graduação em Odontologia proporcionam contato mútuo entre aluno e população, com o intuito de estabelecer um atendimento dentro da realidade socioeconômica dos pacientes e permitir que o estudante signifique a teoria na prática clínica ${ }^{1}$.
A percepção dos usuários a respeito da qualidade do serviço é importante para desenvolver ações e melhorias, tanto por parte do gestor para poder melhorar suas estratégias e ações, como por parte dos próprios profissionais, que estarão mais preparados para entender as expectativas dos usuários ao compreender suas percepções sobre a 
qualidade do atendimento prestado ${ }^{2}$.

A satisfação do paciente é alcançada com a implantação de uma política educacional que considere professores e alunos conscientes e engajados em um processo de transformação social do homem que mostre a ética odontológica e que considere, com dignidade, o real compromisso que o profissional de Odontologia deve ter com a sociedade brasileira ${ }^{3}$.

Além disso, a condição socioeconômica é fator determinante de saúde e de expectativa de vida e há evidências sobre a relação direta entre condições de saúde bucal e perfil socioeconômico ${ }^{4}$. O desequilíbrio na distribuição de renda, aliada à dificuldade dos serviços públicos de saúde em suprir a crescente demanda de pacientes em busca de atendimento odontológico, gera um grande aumento na procura pelas clínicas das instituições de ensino ${ }^{5}$.

Sendo assim, o objetivo desta pesquisa foi avaliar o perfil socioeconômico e a satisfação dos pacientes em relação aos serviços prestados na Clínica de Endodontia de uma instituição de ensino de pós-graduação.

\section{METODOLOGIA}

Trata-se de um estudo observacional transversal em que a coleta de dados foi realizada com pacientes atendidos na Clínica de Endodontia da Associação Brasileira de Odontologia, Secção Piauí (ABO-PI).

A pesquisa foi previamente aprovada pelo Comitê de Ética em Pesquisa da Universidade Federal do Piauí - Campus Ministro Petrônio (CAAE 86460318.2.0000.5214, parecer 2.573.968). Os princípios regidos pelas normas nacionais e internacionais que regulam as pesquisas com seres humanos foram seguidos, respeitando as determinações da resolução 466/12 do Conselho Nacional de Saúde, garantindo a confidencialidade, anonimato e não utilização das informações em prejuízo dos indivíduos.
Os dados foram coletados por três pesquisadores devidamente treinados e calibrados, por meio de questionário estruturado e autoaplicável baseado em instrumentos adotados por estudos anteriores ${ }^{6,7}$, com questões fechadas e abertas, mediante assinatura do Termo de Consentimento Livre e Esclarecido pelos participantes.

Foi realizado um estudo piloto para avaliação prévia do questionário e procedimentos da pesquisa com 10 pacientes selecionados aleatoriamente e que não foram incluídos na amostra final. Após o estudo piloto e as modificações sugeridas e realizadas, o questionário modificado foi distribuído. Os participantes foram orientados pelos pesquisadores a preencher os questionários livremente, sem a intervenção destes, com total autonomia, inclusive para desistir sem incorrer em prejuízo no seu tratamento, sem provocar nenhum dano na sua relação com o profissional em virtude do caráter sigiloso dos resultados obtidos, embora tenha sido ressaltado que o estudo trará uma grande contribuição no sentido de melhorar o atendimento desses pacientes.

Os formulários foram distribuídos a 50 pacientes, aleatoriamente, após o atendimento para aqueles que finalizaram o tratamento e recolhidos após serem respondidos. $\mathrm{O}$ questionário era composto por 22 questões em que foram solicitados dados de identificação como sexo, idade, renda familiar, escolaridade, motivo da procura por atendimento, cujo objetivo foi traçar o perfil socioeconômico do usuário; além de dados referentes à satisfação com o funcionamento da clínica, como confiança, compromisso, empatia, receptividade, autonomia e resolutividade. Foram solicitadas também a opinião sobre a estrutura do ambiente da clínica e questões sobre a satisfação com os aspectos técnicos do atendimento.

Para avaliar a satisfação ao final do tratamento foram utilizadas escalas dimensionais e bipolares, com escores atribuídos entre as 
extremidades. As notas dadas aos diferentes aspectos do tratamento variaram de 0 a 10 , sendo o valor atribuído pelo paciente de forma subjetiva, caracterizando o seu grau de satisfação com o tratamento endodôntico recebido. O cutoff adotado foi o valor 7. Valores menores ou iguais a 7 foram convencionados como insatisfação e valores acima de 7 como satisfatórios.

Os critérios de inclusão adotados no estudo para composição da amostra foram: tratamento endodôntico indicado e finalizado na clínica de endodontia da ABO-PI e ter 18 anos ou mais.

Os resultados foram catalogados e submetidos à análise descritiva da distribuição das frequências absolutas e relativas por meio de tabelas com número, percentual, média e valores mínimos e máximos. Para comparar dados foram aplicados os testes U de Mann-Whitney e de Kruskal-Wallis. Para a análise inferencial das variáveis qualitativas foi aplicado o Teste do Quiquadrado de Pearson. Quando não atendidos os pressupostos deste, nos casos em que as frequências observadas foram menores que 5 , foi realizado o Teste Exato de Fisher. Para isso, as variáveis "satisfação ao final do tratamento" foram transformadas em categóricas e dicotomizadas, obtendo os escores "satisfeito com o tratamento" e "insatisfeito com o tratamento".

O nível de significância foi fixado em $5 \%$. O software PASW-20® (IBM Analytics, Armonk, NY, EUA) foi utilizado para análise estatística a partir da criação de um banco de dados.

\section{RESULTADOS}

A partir da estatística descritiva, foi possível identificar as variáveis relacionadas às características sociodemográficas, à experiência de dor antes do tratamento, à qualidade do atendimento prestado pelo aluno e professor, sugestões para melhoria do atendimento, ao motivo da procura pelo serviço, a informações sobre a organização e infraestrutura da instituição, à indicação da clínica para outros usuários e à satisfação com atendimento recebido.

A média de idade dos pacientes incluídos nesta pesquisa foi de $38,1( \pm 11,9)$ anos, com mínima de 18 e máxima de 66 anos, mais concentrados na faixa etária acima de 35 anos. A maioria era do sexo feminino $(n=41 ; 82,0 \%)$, casada $(n=31 ; 62,0 \%)$, autodeclarada parda $(n=29$; $58,0 \%)$, com ensino médio completo $(n=17$; $34,0 \%)$, vínculo empregatício atual $(n=32 ; 64,0 \%)$, renda familiar mensal de 1 a 2 salários mínimos $(\mathrm{n}=22 ; 44,0 \%)$ (salário mínimo vigente: $\mathrm{R} \$$ 1.045,00), que residiam em Teresina ( $n=44$; $88,0 \%)$ e utilizavam ônibus como meio de transporte $(n=21 ; 42,0 \%)$.

Quanto à experiência da sensação dolorosa, a maioria dos pacientes não estava sentindo dor antes do tratamento realizado $(n=34 ; 68,0 \%)$. Entretanto, 70,6\% $(\mathrm{n}=48)$ haviam recebido alguma informação sobre a necessidade de tratamento. Dos que relataram que estavam sentindo dor $32,0 \%$ ( $n=16$ ), a média de duração foi 30,7 $\pm 28,6$ dias, com no mínimo 3 e máximo 90 dias. A intensidade da dor foi classificada como moderada pela maior parte ( $\mathrm{n}=7 ; 43,7 \%)$, com média de $6,3 \pm 2,3 \mathrm{em}$ uma escala de 0 a 10 . O uso de medicamentos para controle da dor foi realizado por $81,2 \%$ dos pacientes $(n=13)$, sendo a Dipirona $38,5 \%(n=5)$ a mais utilizada.

A maior parte dos usuários $(\mathrm{n}=27 ; 54,0 \%)$ procurou o atendimento por indicação de colegas e amigos. O atendimento foi considerado "ótimo" quando realizado tanto pelo aluno $(\mathrm{n}=39 ; 78,0 \%)$ quanto pelo professor $(\mathrm{n}=40 ; 80 \%)$, com média de $9,4 \pm 0,9$ em uma escala de 0 a 10 . A infraestrutura foi considerada confortável por 86,0\% $(n=43)$, todos recomendariam a instituição $(n=50 ; 100,0 \%)$ e as sugestões mais citadas foram "nenhuma mudança" ( $\mathrm{n}=19 ; 38,0 \%)$, "ser atendido mais vezes na semana" ( $\mathrm{n}=12 ; 24,0 \%)$ e "reduzir o tempo de espera na recepção" $(n=6,12,0 \%)$.

Quanto à satisfação do usuário ao final do 
tratamento endodôntico realizado, a menor nota foi atribuída ao fator "sensação dolorosa" $(6,9 \pm 2,8)$, indicando insatisfação do usuário nesse quesito, sendo as demais notas indicativas de satisfação com o tratamento, o que também é demonstrado com a maior média geral para as notas ao fator "satisfação geral ao final do tratamento" $(8,9 \pm 2,1)$. Pode-se observar ainda que os desvios-padrão foram relativamente baixos, sinalizando uma concordância entre os usuários para os questionamentos levantados para a satisfação com o tratamento (tabela 1).

Tabela 1. Médias, desvio-padrão e valores mínimos e máximos das médias do grau de satisfação ao final do tratamento endodôntico relatado pelos pacientes atendidos na Clínica de Endodontia da ABO-PI

\begin{tabular}{lccc}
\hline \multicolumn{1}{c}{ Variável } & Média (desvio-padrão) & $\begin{array}{c}\text { Valor } \\
\text { mínimo }\end{array}$ & $\begin{array}{c}\text { Valor } \\
\text { máximo }\end{array}$ \\
\hline Tempo de tratamento & $7,5(2,6)$ & 0 & 10 \\
Sensação dolorosa & $6,92,8)$ & 0 & 10 \\
Conforto durante o tratamento & $8,0(2,6)$ & 0 & 10 \\
Conforto com a mastigação ao final do & $8,3(2,3)$ & 0 & 10 \\
tratamento & & & \\
Satisfação com a estética obtida & $8,5(2,2)$ & 0 & 10 \\
Satisfação geral ao final do tratamento & $8,9(2,1)$ & 0 & 10 \\
\hline
\end{tabular}

Foram verificadas associações significativas entre a satisfação geral ao final do tratamento com $\mathrm{o}$ atendimento realizado pelo professor $(\mathrm{p}=0,048$; Teste exato de Fisher), em que a maior parte $(n=30$; $76,0 \%$ ) considerou ótimo e estava satisfeita com o atendimento. Não foram verificadas associações significativas de interesse com as demais variáveis $(\mathrm{p}>0,05)$.

Foram investigadas associações estatisticamente significativas para as variáveis que possivelmente relacionassem o grau de insatisfação/satisfação dos pacientes antes, durante e/ou após o tratamento endodôntico (tabela 2). Mulheres ficaram mais satisfeitas com a estética final obtida $(p=0,017)$. A faixa etária acima de 35 anos ficou mais satisfeita com o atendimento recebido $(\mathrm{p}=0,020)$. Os pacientes que consideraram o ambiente "confortável" ficaram mais satisfeitos com o atendimento recebido $(\mathrm{p}=0,029)$. Pacientes com o ensino médio completo ficaram mais satisfeitos com a estética final obtida $(\mathrm{p}=0,013)$.
Não foram verificadas associações significativas de interesse com as demais variáveis $(\mathrm{p}>0,05)$.

\section{DISCUSSÃO}

Por meio de dados provenientes de um estudo transversal foram analisados o perfil socioeconômico e a satisfação dos usuários dos serviços prestados na Clínica de Endodontia da ABO-PI.Um primeiro aspecto a ser destacado a partir dos resultados deste estudo foi o elevado grau de satisfação indicado por usuários em relação ao serviço odontológico investigado, pois a maioria não sugeriu mudanças no atendimento e todos recomendariam a instituição de ensino. Este achado corrobora as pesquisas de satisfação encontradas na literatura nacional $e$ internacional ${ }^{8,9,10}$. No entanto esses resultados devem ser analisados com cautela, uma vez que, usuários de saúde brasileiros tendem a expor certo conformismo em relação aos serviços ${ }^{11}$, baixo teor crítico e reivindicativo ${ }^{11,12}$ e ainda, uma visão 
equivocada no reconhecimento do serviço de saúde podendo levar a uma satisfação incoerente dos como benefício e não um como um direito, serviços de saúde pesquisados ${ }^{12}$.

Tabela 2. Distribuição e valor de $\mathrm{p}$ das variáveis que possivelmente relacionam o grau de insatisfação/satisfação antes, durante e/ou após o tratamento endodôntico

\begin{tabular}{|c|c|c|c|}
\hline Variáveis & & & $\mathbf{p}^{*}$ \\
\hline \multirow[t]{3}{*}{ Satisfação com a estética obtida } & Gênero & n(\%) & \multirow{3}{*}{$0,017^{\mathrm{a}}$} \\
\hline & Masculino & $9(18,0 \%)$ & \\
\hline & Feminino & $41(82,0 \%)$ & \\
\hline \multirow[t]{5}{*}{ Nota geral pelo atendimento } & Idade (em anos) & n (\%) & \\
\hline & Até 25 & $9(18 \%)$ & \multirow{4}{*}{$\mathbf{0 , 0 2 0} 0^{b}$} \\
\hline & Entre 25 até 35 & $13(26 \%)$ & \\
\hline & Entre 35 até 50 & $14(28,0 \%)$ & \\
\hline & Entre 45 até 70 & $14(28,0 \%)$ & \\
\hline \multirow[t]{4}{*}{ Nota geral pelo atendimento } & Infraestrutura & $\mathrm{n}(\%)$ & \\
\hline & Confortável & $43(86,0 \%)$ & \multirow{3}{*}{$\mathbf{0 , 0 2 9}{ }^{b}$} \\
\hline & Desconfortável & $6(12,0 \%)$ & \\
\hline & Muito quente & $1(2,0 \%)$ & \\
\hline \multirow[t]{8}{*}{ Satisfação com a estética obtida } & Escolaridade & n (\%) & \\
\hline & Fundamental incompleto & $3(6,0 \%)$ & \multirow{7}{*}{$\mathbf{0 , 0 1 3}{ }^{b}$} \\
\hline & Fundamental completo & $1(2,0 \%)$ & \\
\hline & Ensino médio incompleto & $4(8,0 \%)$ & \\
\hline & Ensino médio completo & $17(34,0 \%)$ & \\
\hline & Curso superior incompleto & $7(14 \%)$ & \\
\hline & Curso superior completo & $9(18,0 \%)$ & \\
\hline & Pós-graduação & $9(18,0 \%)$ & \\
\hline
\end{tabular}

a: Teste U de Mann-Whitney; b: Teste de Kruskal-Wallis; *: Diferença estatisticamente significativa

O fator socioeconômico influencia o grau de satisfação do paciente atendido em instituiçãoescola, como revela o estudo de Mascarenhas $(2001)^{13}$, em que o baixo custo do tratamento faz com que os usuários apresentem baixa expectativa em relação ao tratamento ou sejam mais tolerantes. O simples fato de serem atendidos já pode produzir satisfação. Há uma tendência por parte dos usuários de classes sociais menos favorecidas em avaliar positivamente os serviços que lhes são $\operatorname{prestados}^{14,15}$, e esse aspecto foi observado nesta pesquisa.

A maior parte dos participantes deste estudo foram predominantemente do sexo feminino e ficaram mais satisfeitas com a estética final obtida $(\mathrm{p}=0,017)$. O fato de as mulheres estarem mais presentes nas consultas odontológicas se dá pela importância que elas atribuem à saúde bucal, estética e beleza ${ }^{16}$. Quanto à idade houve predominância de indivíduos acima da terceira década de vida. A maior procura vinculada a essa faixa etária pode estar associada a uma maior predominância de problemas bucais, principalmente periodontais ${ }^{17}$.

A boa comunicação profissional/paciente, incluindo a transmissão apropriada de informações 
sobre o tratamento e a consequente adequação da expectativa do paciente aos resultados esperados, é de extrema importância para o êxito de qualquer trabalho $^{18,19}$. É interessante ressaltar que o atendimento ofertado tanto pelo aluno quanto pelo professor foi considerado "ótimo", sendo esse dado de fundamental importância para uma autoanálise da qualidade e que o atendimento na Clínica de Endodontia da ABO-PI corresponde à expectativa dos usuários.

Os pacientes atendidos consideraram a Clínica de Endodontia como "confortável" e atribuíram melhor nota geral ao atendimento $(\mathrm{p}=0,029)$. Os dados obtidos nesta pesquisa corroboram com resultados encontrados na literatura em que o ambiente é definido como um elemento que determina a satisfação do usuário. Os pacientes atendidos demonstram uma opinião positiva a respeito da organização, limpeza, conforto e iluminação das clínicas ${ }^{20-23}$. Um fator recorrente de insatisfação entre usuários e profissionais da saúde é a precariedade da estrutura física das unidades de saúde ${ }^{9,24}$.

Iniciativas de reorganização dos processos de trabalho, assim como otimização dos fluxos internos podem contribuir demasiadamente para a melhoria da qualidade dos serviços e consequentemente na satisfação dos usuários, devendo, dessa forma, a organização dos serviços e das práticas de saúde ser capaz de realizar uma apreensão ampliada das necessidades da população sob a sua responsabilidade ${ }^{12}$.

O corte transversal com coleta em questionários autoaplicáveis possibilita a ocorrência de viés de memória e influência de fatores locais e isso pode ser visto como uma limitação do presente estudo porque impede hipótese de causalidade. Além disso, o viés de informação também pode ter influenciado os resultados, pois os pacientes podem ter relatado percepção favorável por medo de não conseguirem mais atendimento na instituição.
No entanto, estudos transversais são úteis para identificar possíveis fatores associados com a insatisfação dos usuários com o tratamento endodôntico que podem ser investigados em outras avaliações como fatores associados definitivos. Foi utilizada uma amostra intencional devido ao intenso fluxo de pacientes e pela dificuldade em se realizar o cálculo amostral sem ter uma margem da quantidade total de pacientes que frequentam a instituição de pós-graduação. Porém, a seleção dos participantes foi de forma aleatória, no intuito de evitar viés de seleção.

O ponto forte desta pesquisa está na investigação de possíveis fatores relacionados com a insatisfação com o atendimento fornecido e conhecimento do perfil socioeconômico do usuário. Isso pode fornecer subsídios para que o serviço de saúde acesse as percepções e expectativas manifestadas pelos usuários como "ser atendido mais vezes na semana" e "reduzir o tempo de espera na recepção", além de prover informações em quantidade e qualidade afim de alcançar maior satisfação e possibilidade de intervir em melhorias nas esferas que possam interferir nesse fator.

\section{CONCLUSÕES}

De acordo com a metodologia empregada, conclui-se que o perfil socioeconômico dos pacientes atendidos na Clínica de Endodontia ABO-PI contempla, em sua maioria, mulheres casadas, autodeclaradas pardas, acima da terceira década de vida, com vínculo empregatício e baixa renda familiar, com ensino médio completo, residentes na capital e que usam ônibus e carro próprio como meio de transporte, reforçando o papel social da instituição-escola. De uma maneira geral, a qualidade no atendimento dos serviços considerada pelos usuários como fornecedora de um bom atendimento, tanto no aspecto técnico como na relação profissional-paciente. 


\section{ABSTRACT \\ Socio-economic profile and level of satisfaction of patients seen at the endodontics clinic of a postgraduate institution}

This study aimed to assess the socio-economic profile and patient satisfaction with the services provided at the Endodontics Clinic of a postgraduate teaching institution. Data collection was carried out in cross-section through a structured and self-administered questionnaire. The sample was intentional and random $(n=50)$ for users who had completed endodontic treatment. A dimensional and bipolar scale was used to evaluate satisfaction at the end of the treatment with scores attributed between the extremities. The scores given to the different aspects of the treatment ranged from 0 to 10 with a cutoff of 7 . The results were cataloged and submitted to descriptive and inferential analysis with a significance level set at 5\%. The socioeconomic profile of the patients treated includes, in its majority, married, brown women, above the third decade of life, with employment and low family income, with complete high school education, residing in the capital, and who use buses and their own car as a means of transport. The service was considered satisfactory. Users seek care more by referral from other individuals than by their financial condition, are satisfied with the care provided by the student and the teacher, and consider the infrastructure of the institution comfortable. There are still aspects that need to be improved, especially concerning waiting and service times and the possibility of attending more than once a week.

Descriptors: Patient Satisfaction. Evaluation of Health Services. Academic Institutions. Endodontics.

\section{REFERÊNCIAS}

1. Castro JDB, Silva VB. Satisfação no atendimento odontológico: um estudo na $\mathrm{COE}$ (Clínica Odontológica de Ensino) de Anápolis - Unievengélica. Rev Administra-Ação. 2008; 5:34-44.

2. Gonçalves EV, Verdi MIM. Os problemas éticos no atendimento a pacientes na clínica odontológica de ensino. Ciênc Saúde Coletiva. 2007; 12(3):755-64.

3. Vomero MF. Entendendo a relação paciente/profissional. Rev Reg Araçatuba (Assoc Paul Cir Dent). 2000; 54(4):267-78.

4. Stamm AMNF, Osellame R, Duarte F, Cecato F, Medeiros LA, Marasciulo AC. Perfil socioeconômico dos pacientes atendidos no ambulatório de Medicina interna do Hospital universitário da UFSC. ACM Arq Catarin Med. 2002; 31(1-2):17-24.

5. Tiedman CR, Linhares E, Silveira JLGC. Clínica Integrada Odontológica: perfil e expectativas dos usuários e alunos. Pesq Bras Odontoped Clin Integr. 2005; 5(1):53-8.

6. Ribeiro ILA, Veloso HHP, Valença AMG, Lima Neto EA, Brasil Júnior O. Avaliação da qualidade de vida e nível de satisfação do usuário da rede de atenção especializada com o tratamento endodôntico no município de João Pessoa, Paraíba, Brasil, 2009. Rev Odontol Bras Central. 2012; 21(59):557-63.

7. Pompeu JGF, Carvalho ILM, Pereira JA, Cruz Neto RG, Prado VLG, da Silva CLV. Avaliação do nível de satisfação dos usuários atendidos na clínica integrada do curso de odontologia da Faculdade Novafapi em Teresina (PI). Odontol Clín-Cient. 2012; 11(1):31-6.

8. Goetz K, Szecsenyi J, Klingenberg A, Brodowski M, Wensing M, Campbell SM. Evaluation of patient perspective on quality of oral health care in Germany - an exploratory study. Int Dent J. 2013; 63(6):317-323.

9. Sobreira PGP, Vasconcellos MTL, Portela MC. Avaliação do processo de aconselhamento pré-teste nos Centros de Testagem e Aconselhamento (CTA) no Estado do Rio de Janeiro: a percepção dos usuários e profissionais de saúde. Ciênc Saúde Coletiva. 2012; 17(11):3099-113. 
10. Priporas CV, Laspa C, Kamenidou I. Patient satisfaction measurement for in-hospital services: a pilot study in Greece. J Med Mark. 2008; 8(4):325-40.

11. Serapioni M, Silva MGC. Evaluation of the quality of Family Healthcare program in municipalities of Ceará: a multidimensional approach. Ciênc Saúde Coletiva. 2011; 16(11):4315-26.

12. Brandão ALRBS, Giovanella L, Campos CEA. Avaliação da atenção básica pela perspectiva dos usuários: adaptação do instrumento EUROPEP para grandes centros urbanos brasileiros. Ciênc Saúde Coletiva. 2013; 18(1):103-14.

13. Mascarenhas AK. Patient satisfaction with the comprehensive care model of dental care delivery. J Den Educ. 2001; 65(11):1266-71.

14. Vaitsman J, Andrade GRB. Satisfação e responsividade: formas de medir a qualidade e a humanização da assistência à saúde. Ciênc Saúde Coletiva. 2005; 10(3):599-613.

15. Lemme AC, Noronha G, Resende JB. A Satisfação do usuário em hospital universitário. Rev Saúde Públ. 1991; 25(1):416.

16. Carvalho G, Rosemburg CP, Buralli KO. Avaliação de ações e serviços de saúde. Mundo Saúde. 2000; 24(1):72-8.

17. Brasil. Ministério da Saúde. Projeto SB 2003. [Acesso em: 25 de abril, 2020]. Disponível em: https://bvsms.saude.gov.br/bvs/publicacoes/c ondicoes_saude bucal.pdf.

18. Garbin CA, Garbin AJI, Dossi SP, Macedo L, Macedo V. O tratamento odontológico: informações transmitidas aos pacientes e motivos de insatisfação. Rev Odontol UNESP. 2008; 37(2):177-81.

19. Kruse AB, Heil HK, Struß N, Fabry G, Silbernagel W, Vach K, Ratka-Krüger $\mathrm{P}$, Woelber JP. Working experience is not a predictor of good communication: Results from a controlled trial with simulated patients. Eur J Dent Educ. 2020; 24(2):177-85.

20. Leão ATT, Dias K. Avaliação dos serviços de saúde prestados por faculdades de odontologia: a visão do usuário. Rev Bras Odontol Saúde Coletiva. 2001; 2(1):40-6.

21. Ramos FB. Como o paciente se sente ao ser atendido por um aluno de odontologia? Rev CROMG. 2001; 7(1):10-5.

22. Ramos FB. Eficácia do atendimento oferecido aos pacientes da clínica integrada da Faculdade Federal de Odontologia de Diamantina. Rev CROMG. 1997; 3(2):56-63.

23. Coelho IV, Melo ARF, Caetano RM, Silva CLM, Habibe RCH, Habibe CH. Avaliação da satisfação do paciente atendido na Clínica Integrada Odontológica do UniFOA. Braz J Hea Rev. 2020; 3(1):673-83.

24. Nora CRD, Jungles JR. Política de humanização na atenção básica: revisão sistemática. Rev Saúde Publica 2013; 47(6):1186-200.

\section{Correspondência para:}

Markelane Santana Silva e-mail: markelanesantanasilva@gmail.com

Rua José Amâncio de Assunção, 120

64260-000 Piripiri/PI 